\title{
Efficacy of Plasmapheresis in Nivolumab-Associated ANCA Glomerulonephritis: A Case Report and Pathophysiology Discussion
}

\author{
Reda Laamech ${ }^{a} \quad$ Florian Terrec $^{a} \quad$ Camille Emprou $^{b}$ \\ Anne Claire Toffart ${ }^{c, d}$ Thomas Pierret ${ }^{c}$ Hamza Naciri-Bennani ${ }^{a}$ \\ Lionel Rostaing a, d Johan Noble ${ }^{a} d$ \\ aNephrology, Hemodialysis, Apheresis and Kidney Transplantation Department, University \\ Hospital Grenoble, La Tronche, France; bPathology Department, University Hospital \\ Grenoble, La Tronche, France; Thoracic Oncology Unit, University Hospital Grenoble, \\ La Tronche, France; ${ }^{\mathrm{d} G r e n o b l e ~ A l p e s ~ U n i v e r s i t y, ~ G r e n o b l e, ~ F r a n c e ~}$
}

\section{Keywords}

Nivolumab · Plasmapheresis · ANCA vasculitis · Kidney failure

\begin{abstract}
Immune checkpoint inhibitors (ICls) have revolutionized solid organ and hematologic cancer treatments by improving overall prognoses. However, they can lead to overactivation of the immune system and several immune-related adverse events and sometimes affecting the renal system. Although acute interstitial nephritis is well described, we know little about ICl-associated glomerular injury. Herein, we report an exceptional case of renal ANCA positive-associated vasculitis (AAV) after nivolumab therapy. Three weeks after the last nivolumab injection, the patient presented with proteinuria at $1.73 \mathrm{~g} / \mathrm{g}$ of creatininuria, hematuria, and acute kidney injury needing dialysis associated with lung hemorrhage; anti-neutrophil cytoplasmic antibody (ANCA titer $\geq 1,280$ with myeloperoxidase specificity of $780 \mathrm{U} / \mathrm{mL}$ ) was positive, and kidney biopsy confirmed glomerular injury with crescents. The patient underwent treatment with steroid pulses, rituximab, and plasmapheresis, resulting in an improvement of the renal function and lung hemorrhage and produced a negative ANCA titer. Despite the results of the PEXIVAS study and the absence of clear benefit of plasmapheresis demonstrated in idiopathic AAV, we suggest that drug-induced AAV may be effectively treated by plasmapheresis, steroids, and rituximab.
\end{abstract}

(C) 2021 The Author(s).

Published by S. Karger AG, Basel 


\section{Introduction}

Immune checkpoint inhibitors (ICIs) have greatly improved the overall prognosis for several malignancies. These monoclonal antibodies act by blocking immune checkpoints that modulate the activation of lymphocytes.

Three checkpoint proteins are responsible for inducing anergy: cytotoxic T-lymphocyteassociated antigen 4 (CTLA4), the programmed death-1 receptor (PD-1), and its ligand PD-L1. These molecules are localized on several immune system cells, such as T cells, B cells, and dendritic cells. PD-1 regulates cytotoxic T-cell proliferation and B-cell overall survival, whereas CTLA4 mostly inhibits memory T-cell activation [1].

Tumor cells, in order to escape the immune system, decrease the expression of tumorassociated antigens and major histocompatibility complex molecules: they secrete immunosuppressive molecules and selectively upregulate checkpoint inhibitor receptor molecules in their microenvironment. Understanding these mechanisms has permitted to specifically target this pathway to treat several malignancies using ICIs.

However, upregulating the immune response may result in immune-related adverse events. ICIs may cause autoimmune diseases due to tissue-specific self-reactive T-cell activation. The most frequent organs affected by ICI-induced autoimmune diseases are the skin, the endocrine system, and the gastrointestinal tract. Yet, adverse events related to the kidneys can also occur [2,3]. Acute tubulointerstitial nephritis is the most common pathological finding [4, 5]. To date, very few cases of ICI-associated glomerulonephritis have been described, and its specific management and treatment remains unknown [6, 7]. Herein, we report on a case of renal ANCA-associated vasculitis (AAV) associated with nivolumab given to a patient presenting with metastatic non-small-cell lung carcinoma.

\section{Case Presentation}

An 81-year-old man was diagnosed with metastatic non-small-cell lung adenocarcinoma in May 2018. The initial treatment was cisplatin and pemetrexed from May 2018 to July 2018 and then pemetrexed alone from July 2018 to September 2019. Remission occurred from September 2019 to February 2020. Then, in February, the tumor mass has increased on chest CT-scan. He was placed on nivolumab therapy ( 240 mg every 2 weeks); the last dose was given on April 28, 2020. Nivolumab was then discontinued because of asthenia. Kidney function was normal at that time, i.e., serum creatinine (Scr) was $1.09 \mathrm{mg} / \mathrm{dL}$ and estimated glomerular filtration rate (CKD-EPI) was $64 \mathrm{~mL} / \mathrm{min} / 1.73 \mathrm{~m}^{2}$. There was no microscopic hematuria, and proteinuria level was $0.6 \mathrm{~g} / \mathrm{L}$.

At 3 weeks after nivolumab was discontinued, the patient presented with fatigue, fever, and shortness of breath that needed oxygen therapy because of a lung hemorrhage. Scr level had increased to $5.66 \mathrm{mg} / \mathrm{dL}$. Proteinuria was $1.73 \mathrm{~g} / \mathrm{g}$ of creatininuria with a glomerular profile ( $46 \%$ of albumin) associated with hematuria. Anti-neutrophil cytoplasmic antibodies (ANCA) were positive with a titer $\geq 1 / 1,280$ (indirect immunofluorescence) with a myeloperoxidase (MPO) specificity of $780 \mathrm{U} / \mathrm{mL}$ (enzyme immunoassay, $N<5 \mathrm{U} / \mathrm{mL}$ ).

A kidney biopsy, shown in Figure 1, performed on June 8, revealed crescentic glomerulonephritis. There were 23 glomeruli. Cellular to fibrocellular crescents involved $78 \%$ of glomeruli, and $22 \%$ of glomeruli were globally sclerosed. Fibrinoid necrosis involved $52 \%$ of glomeruli, and slight fibrosis surrounding rare atrophic tubules (5\%) was present. Immunofluorescence staining was negative. Kidney failure worsened, and hemodialysis was initiated on June 11. On the day of hemodialysis initiation, nivolumab trough level was $4.1 \mu \mathrm{g} / \mathrm{mL}$. The patient had no other organ injury apart from renal and lung involvement.

\section{Karger'}


Fig. 1. Renal biopsy (trichrome stain) showing the patient's crescentic glomerulonephritis. Bowman's space is occupied by layers of proliferating epithelial cells forming a crescent. Black arrow shows the extracapillary proliferation.
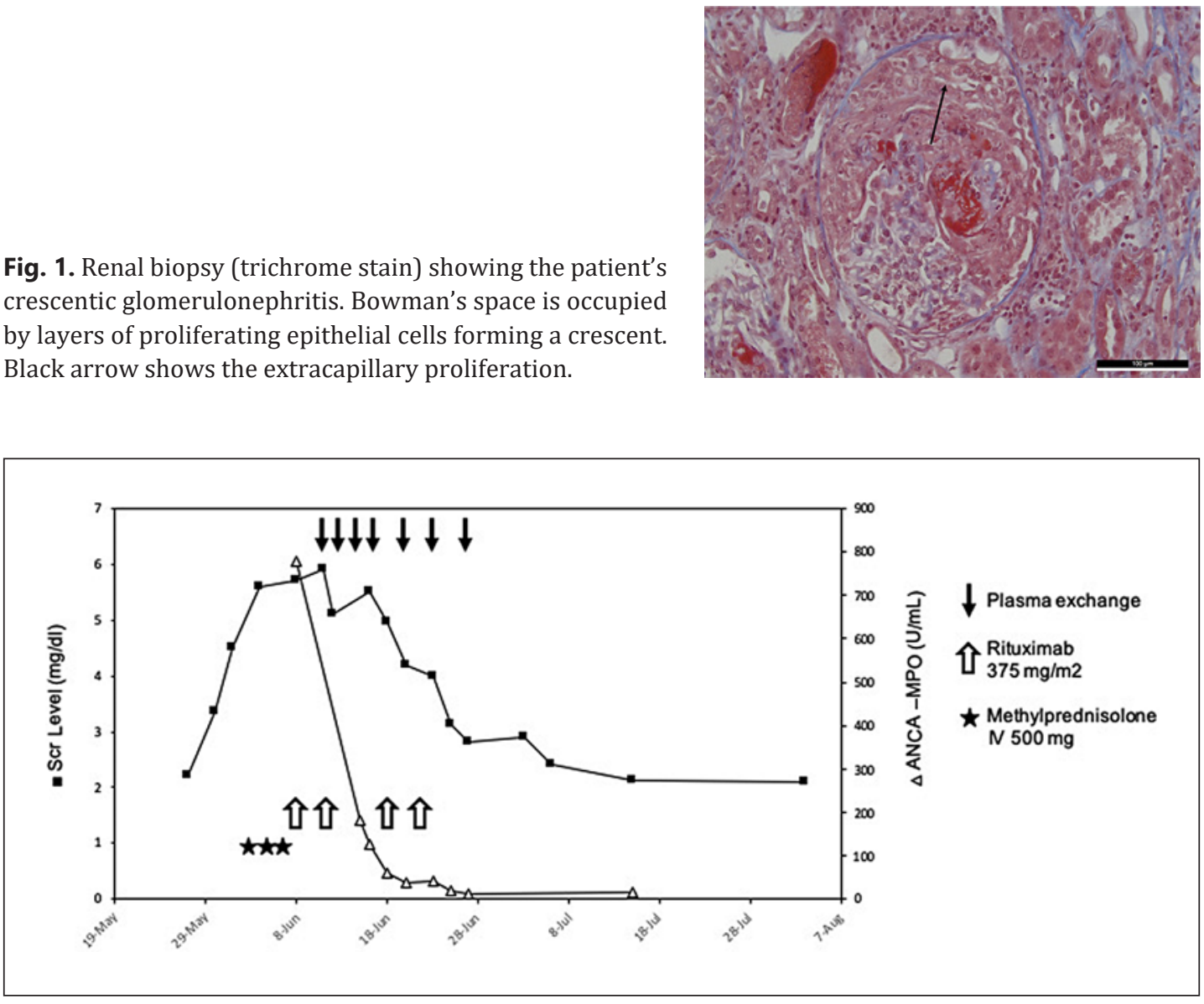

Fig. 2. Outcome of serum creatinine (milligrams per deciliter) and ANCA titers before and after treatment by steroids, rituximab, and plasmapheresis.

As of June 4, the patient received 3 methylprednisolone (MP) pluses (500 mg), followed by oral prednisolone $\left(1 \mathrm{mg} / \mathrm{kg}\right.$ ), and 4 perfusions of rituximab at $375 \mathrm{mg} / \mathrm{m}^{2}$ on June 8,14 , 20 , and 27. Because there was no renal response after MP pulses, we decided to start plasmapheresis: 7 sessions were performed between June 13 and 27. Scr levels and anti-MPO titers under treatment are shown in Figure 2. At the end of plasmapheresis, nivolumab trough level was $<3.0 \mu \mathrm{g} / \mathrm{mL}$.

Kidney function progressively improved allowing hemodialysis to be discontinued by June 25. A chest CT-scan, performed at the end of treatment, did not show any recurrence of the lung hemorrhage. At nearly 1 year, renal function was stable, i.e., estimated glomerular filtration rate at $20 \mathrm{~mL} / \mathrm{min} / 1.73 \mathrm{~m}^{2}$ and proteinuria at $0.6 \mathrm{~g} / \mathrm{L}$. To date, there is no sign of adenocarcinoma worsening, and the patient is still alive.

\section{Discussion and Conclusion}

The incidence of acute kidney injury associated with ICIs occurs in $~ 1.4 \%-4.9 \%$ of cases according to recent reports [4]. The most common pathological disorder reported is acute tubulointerstitial nephritis [8]. Nivolumab has been rarely described to be associated with glomerulopathies: focal and segmental glomerulosclerosis [9], PLA2R-negative membranous 
nephropathy [10], and mesangial expansion with no crescents or endocapillary hypercellularity [11]. Clinical cases and outcomes of ICI-related glomerulopathies reported in the literature are summarized in Table 1 . The temporal association of AAV with the good evolution of the cancer under nivolumab was discordant with the usual time appearance of paraneoplastic AAV described in the literature and made it less plausible than a drug effect [12-14]. Moreover, ICI therapies are associated in the literature with many autoimmune disorders involving many organs including the kidney. To the best of our knowledge, this is the second case of renal ANCA positive AAV induced by nivolumab.

The severity of ICI-related glomerulopathies and responses to treatments differ, partly due to variations between cases. Overall, the variety of ICI-induced renal manifestations suggests multiple complex mechanisms, which should be further elucidated.

A rare case of MPO-ANCA-positive glomerulonephritis, supposedly induced by tremelimumab, was effectively treated with plasmapheresis [15]. Direct involvement of ANCA in the mechanisms of pathogenesis is yet to be proven although animal models clearly indicate the pathogenicity of ANCAs [16].

ANCA stimulates the production of reactive oxygen species and the degranulation of proteolytic enzymes by polymorphonuclear, after pre-stimulation by pro-inflammatory cytokines (TNF-alpha) and adhesion to the endothelium of small vessels. Immunization of genetically MPO-deficient mice with murine MPO and the transfer of murine ANCA-MPO IV in mice without functional lymphocytes (Rag2-/-) has demonstrated systemic AAV, which was prevented by depletion of polymorphonuclear [17].

In our patient, it is possible that the favorable outcome when using plasmapheresis in the setting of ANCA vasculitis-associated severe renal disorders may be explained in several ways: i.e., by the clearance of ANCA and/or clearance of other auto-antibodies and/or removal of circulating microparticles and nonspecific inflammatory molecules (complement system and cytokines) and finally by the removal of the responsible drug (i.e., nivolumab) which may act as a trigger of the autoimmune disease. In our case, the nivolumab dosage was still positive at the time of plasmapheresis initiation. The complement system, and in particular the alternative pathway, is implicated in the development of tissue lesions, and plasmapheresis allows efficient and rapid clearance of those complement proteins [18]. Plasma exchange also removes auto-antibodies, and their resynthesis is progressively slowed by medication.

The efficacy of plasmapheresis in AAV was demonstrated in the MEPEX trial [19]. This study reported that in patients with creatinine $>500 \mu \mathrm{mol} / \mathrm{L}(5.8 \mathrm{mg} / \mathrm{dL})$ at diagnosis of AAV, renal recovery was greater and the need for dialysis reduced after plasma exchange compared to IV MP. All patients of this study received oral prednisolone and oral cyclophosphamide.

Recently, the Plasma Exchange and Glucocorticoids in Severe ANCA-Associated Vasculitis (PEXIVAS) study assessed the efficacy of plasmapheresis in patients with AAV and kidney involvement and/or pulmonary involvement [20]. The authors found no difference in benefit of plasmapheresis versus standard of care (i.e., without plasma exchange) regarding a composite endpoint including mortality or end-stage kidney failure after a median follow-up of 3 years. They concluded that there was no justification for plasma exchange in patients with AAV and kidney or pulmonary involvement, thus questioning the benefit of plasmapheresis for this indication. Yet, many case series report the potential benefit of plasmapheresis in AAV in a subset of patients presenting with alveolar hemorrhage. In 2021, many authors have not abandoned plasmapheresis, and its utilization is still debated in the literature [21, 22].

In this case of ICI-associated ANCA positive AAV, the efficacy of plasmapheresis on outcome led us to discuss the mechanisms of drug-induced AAV and the difference compared to idiopathic AAV $[23,24]$. There may be several hypotheses: (1) nivolumab was directly or indirectly associated with the synthesis of ANCA: therefore, its discontinuation and the natural clearance of antibodies was sufficient to change the course of the disease; (2) nivolumab was

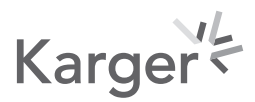


Case Reports

in Nephrology and Dialysis
Case Rep Nephrol Dial 2021;11:376-383 DOI: $10.1159 / 000518304$

(c) 2021 The Author(s). Published by S. Karger AG, Basel www.karger.com/cnd

Laamech et al.: Plasmapheresis for Nivolumab-Induced ANCA Vasculitis

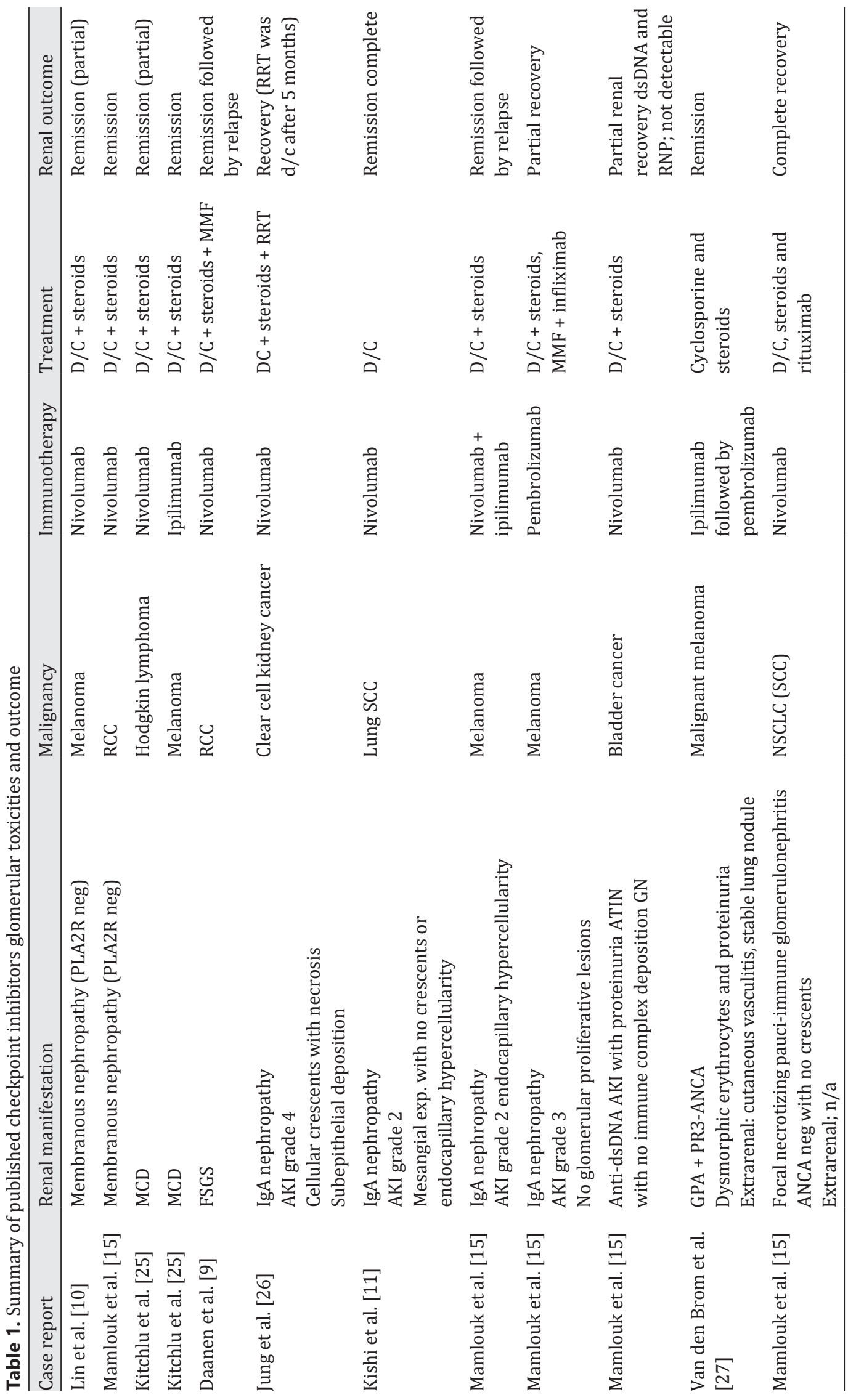


Case Reports

in Nephrology and Dialysis
Case Rep Nephrol Dial 2021;11:376-383 www.karger.com/cnd

Laamech et al.: Plasmapheresis for Nivolumab-Induced ANCA Vasculitis

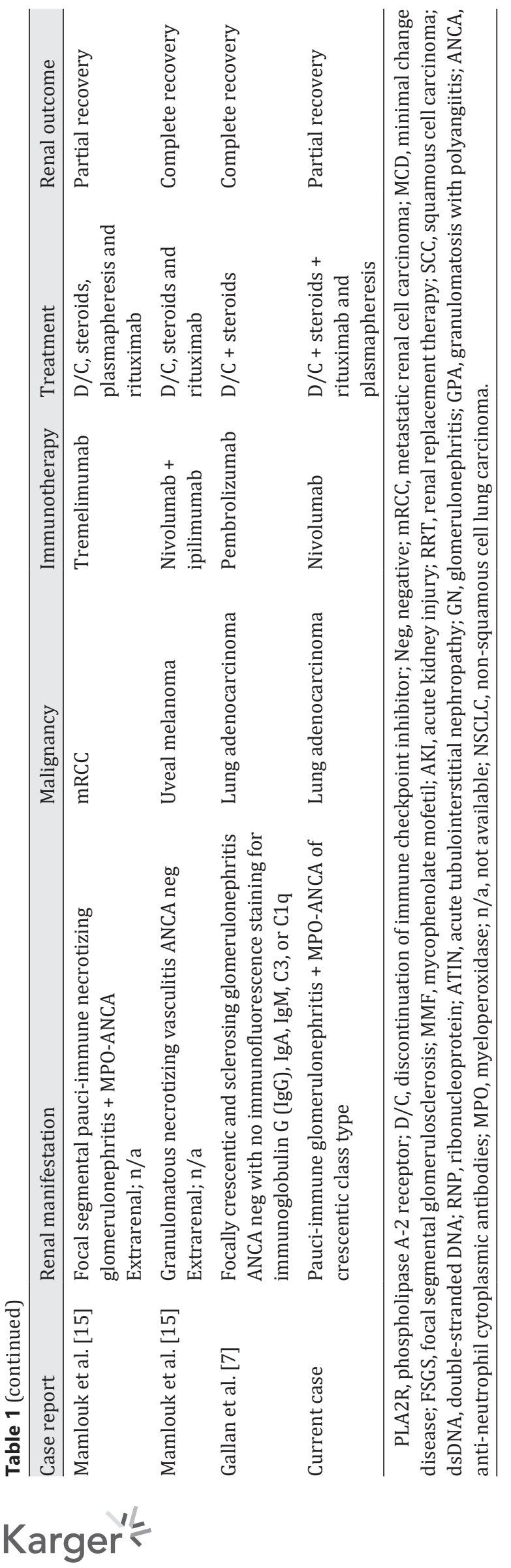


Case Reports

in Nephrology

and Dialysis

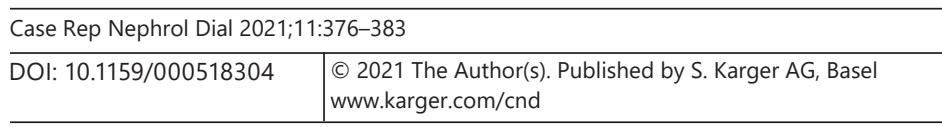

Laamech et al.: Plasmapheresis for Nivolumab-Induced ANCA Vasculitis

associated with the synthesis of ANCA, and plasma exchange helped lowering nivolumab plasma levels and thus ANCA titers; or (3) nivolumab was a trigger for AAV, and the clearance of ANCA and several pro-inflammatory proteins was efficient through plasma exchange. In conclusion, we report the second case of nivolumab-induced ANCA positive AAV. Our finding invites us to investigate the mechanisms involved in the induction of autoimmunity in immunotherapy, including when disease occurs as a rare event. AAVs induced by ICI and associated with renal issues have rarely been described and need to be researched further. Finally, despite the results of the PEXIVAS study, the authors can make the hypothesis that plasmapheresis may be efficient in the subgroup of ICI-induced AAV which will have to be shown in larger studies.

\section{Statement of Ethics}

Written informed consent was obtained from the patient for publication of this case report and any accompanying images. According to the local ethics committee (French CCP "commission de protection des personnes"), no more document is needed. The manuscript was conducted ethically in accordance with the World Medical Association Declaration of Helsinki.

\section{Conflict of Interest Statement}

The authors of this manuscript have no conflicts of interest to disclose.

\section{Funding Sources}

This manuscript did not receive any funding.

\section{Author Contributions}

R.L. wrote the manuscript, J.N. and L.R. supervised and corrected the manuscript, C.E. interpreted the pathology findings, H.N.B. performed plasmapheresis, and F.T., A.C.T., and T.P. were the doctors in charge of the patient. All authors read and approved the manuscript.

\section{Data Availability Statement}

All data generated or analyzed during this study are included in this article. Further enquiries can be directed to the corresponding author.

\section{References}

1 Gremese E, Alivernini S, Ferraccioli ES, Ferraccioli G. Checkpoint inhibitors (CPI) and autoimmune chronic inflammatory diseases (ACIDs): tolerance and loss of tolerance in the occurrence of immuno-rheumatologic manifestations. Clin Immunol. 2020;214:108395.

2 Wanchoo R, Karam S, Uppal NN, Barta VS, Deray G, Devoe C, et al. Adverse renal effects of immune checkpoint inhibitors: a narrative review. Am J Nephrol. 2017;45(2):160-9.

3 Perazella MA, Shirali AC. Nephrotoxicity of cancer immunotherapies: past, present and future. J Am Soc Nephrol. 2018;29(8):2039-52. 
4 Cortazar FB, Marrone KA, Troxell ML, Ralto KM, Hoenig MP, Brahmer JR, et al. Clinicopathological features of acute kidney injury associated with immune checkpoint inhibitors. Kidney Int. 2016;90(3):638-47.

5 Shirali AC, Perazella MA, Gettinger S. Association of acute interstitial nephritis with programmed cell death 1 inhibitor therapy in lung cancer patients. Am J Kidney Dis. 2016 Aug;68(2):287-91.

6 Ashour T, Nakhoul G, Patil P, Funchain P, Herlitz L. Immune check point inhibitor-associated glomerulonephritis. Kidney Int Rep. 2019 Feb;4(2):355-9.

7 Gallan AJ, Alexander E, Reid P, Kutuby F, Chang A, Henriksen KJ. Renal vasculitis and pauci-immune glomerulonephritis associated with immune checkpoint inhibitors. Am J Kidney Dis. 2019;74(6):853-6.

8 Koda R, Watanabe H, Tsuchida M, Iino N, Suzuki K, Hasegawa G, et al. Immune checkpoint inhibitor (nivolumab)associated kidney injury and the importance of recognizing concomitant medications known to cause acute tubulointerstitial nephritis: a case report. BMC Nephrol. 2018;19(1):48.

9 Daanen RA, Maas RJH, Koornstra RHT, Steenbergen EJ, van Herpen CML, Willemsen AECAB. Nivolumab-associated nephrotic syndrome in a patient with renal cell carcinoma: a case report. J Immunother. 2017 Dec;40(9):345-8.

10 Lin JS, Wang DY, Mamlouk O, Glass WF, Abdelrahim M, Yee C, et al. Immune checkpoint inhibitor associated reactivation of primary membranous nephropathy responsive to rituximab. J Immunother Cancer. 2020 Oct; 8(2):e001287.

11 Kishi S, Minato M, Saijo A, Murakami N, Tamaki M, Matsuura M, et al. IgA nephropathy after nivolumab therapy for postoperative recurrence of lung squamous cell carcinoma. Intern Med. 2018 May 1;57(9):1259-63.

12 Baschinsky DY, Baker PB, Niemann TH, Wilmer WA. Pauci-immune ANCA-positive crescentic glomerulonephritis associated with metastatic adenocarcinoma of the lung. Am J Kidney Dis. 2000 Oct;36(4):E24.

13 Baldeo C, Ali R, Hritani A, Poenariu A. ANCA-negative pauci-immune crescentic glomerulonephritis linked with non-small cell carcinoma of the lung. Case Rep Nephrol Dial. 2015 Jul 4;5(2):168-72.

14 Morikawa T, Yoshida A, Kobayashi S, Shibata M, Hamada M, Kishida M, et al. AP-VAS 2012 case report: a case of ANCA-negative pauci-immune crescentic glomerulonephritis associated with IL-6-producing adenosquamous cell carcinoma of the lung. CEN Case Rep. 2013 Mar 14;(2):158-64.

15 Mamlouk O, Lin JS, Abdelrahim M, Tchakarov AS, Glass WF, Selamet U, et al. Checkpoint inhibitor-related renal vasculitis and use of rituximab. J Immunother Cancer. 2020 Jul;8(2):e000750.

16 Little MA, Al-Ani B, Ren S, Al-Nuaimi H, Leite M Jr, Alpers CE, et al. Anti-proteinase 3 anti-neutrophil cytoplasm autoantibodies recapitulate systemic vasculitis in mice with a humanized immune system. PLoS One. 2012; $7(1): \mathrm{e} 28626$.

17 Xiao H, Heeringa P, Hu P, Liu Z, Zhao M, Aratani Y, et al. Antineutrophil cytoplasmic autoantibodies specific for myeloperoxidase cause glomerulonephritis and vasculitis in mice. J Clin Invest. 2002 Oct;110(7):955-63.

18 Jennette JC, Falk RJ, Hu P, Xiao H. Pathogenesis of antineutrophil cytoplasmic autoantibody-associated small-vessel vasculitis. Annu Rev Pathol. 2013 Jan 24;8:139-60.

19 Jayne DRW, Gaskin G, Rasmussen N, Abramowicz D, Ferrario F, Guillevin L, et al. Randomized trial of plasma exchange or high-dosage methylprednisolone as adjunctive therapy for severe renal vasculitis. J Am Soc Nephrol. 2007 Jul;18(7):2180-8.

20 Walsh M, Merkel PA, Peh C-A, Szpirt WM, Puéchal X, Fujimoto S, et al. Plasma exchange and glucocorticoids in severe ANCA-associated vasculitis. N Engl J Med. 2020 13;382(7):622-31.

21 Kronbichler A, Shin JI, Wang CS, Szpirt WM, Segelmark M, Tesar V. Plasma exchange in ANCA-associated vasculitis: the pro position. Nephrol Dial Transplant. 2021 Jan 25;36(2):227-31.

22 Specks U, Fussner LA, Cartin-Ceba R, Casal Moura M, Zand L, Fervenza FC. Plasma exchange for the management of ANCA-associated vasculitis: the con position. Nephrol Dial Transplant. 2021 Jan 25;36(2):231-6.

23 Grau RG. Drug-induced vasculitis: new insights and a changing lineup of suspects. Curr Rheumatol Rep. 2015 Dec;17(12):71.

24 Nakazawa D, Masuda S, Tomaru U, Ishizu A. Pathogenesis and therapeutic interventions for ANCA-associated vasculitis. Nat Rev Rheumatol. 2019;15(2):91-101.

25 Kitchlu A, Fingrut W, Avila-Casado C, Chan CT, Crump M, Hogg D, et al. Nephrotic syndrome with cancer immunotherapies: a report of 2 cases. Am J Kidney Dis. 2017;70(4):581-5.

26 Jung K, Zeng X, Bilusic M. Nivolumab-associated acute glomerulonephritis: a case report and literature review. BMC Nephrol. 2016;17(1):188.

27 van den Brom RRH, Abdulahad WH, Rutgers A, Kroesen B-J, Roozendaal C, de Groot DJA, et al. Rapid granulomatosis with polyangiitis induced by immune checkpoint inhibition. Rheumatology. 2016;55(6):1143-5. 